\title{
Design of a New Kossel Pattern Generator
}

\author{
D. L. Vieth and H. Yakowitz
}

\author{
Institute for Materials Research, National Bureau of Standards, Washington, D.C. 20234
}

(August 22, 1967)

\begin{abstract}
A Kossel pattern generator, designed and built at the National Bureau of Standards, is described in detail. The unit is modular and consists of an electron beam column, vacuum system, light microscope, film cassette, and Kossel camera. The camera component includes microgoniometric capabilities. The Kossel $x$-ray technique enables the investigator to obtain lattice spacing data precise to two or three parts per million, and orientation of crystals to $0.1^{\circ}$ of arc. The Kossel pattern generator described permits data for such determinations to be obtained quickly and precisely.
\end{abstract}

Key Words: Divergent x-ray beam, instrument design, Kossel camera, Kossel method, x-ray instrumentation.

\section{Introduction}

A divergent $\mathrm{x}$-ray beam, emanating from an effective point source of $x$-radiation, produces diffraction cones on propagating within a single crystal. These cones have a semiapex angle of $\left(90^{\circ}-\theta\right),(\theta$ the Bragg angle); their directions are governed by the crystal orientation and by the lattice symmetry. When such cones are intercepted by a flat photographic film, conic sections corresponding to each monochromatic component (such as $K \alpha_{1}, K \alpha_{2}$, and $K \beta$ ) of the primary beam will be recorded superimposed upon a general background blackening. These conic sections have been referred to as Kossel lines in honor of that pioneer of the divergent beam $x$-ray technique [1]. ${ }^{1}$ The entire photograph is often called a Kossel pattern, or, if the x-ray source happens to be external to the diffracting crystal, the photograph may be called a pseudo-Kossel pattern [2].

Information which can be obtained from the photograph includes lattice spacings, crystal orientation, and data concerning the degree of crystal perfection. In particular, lattice spacing data precise to two or three parts per million can be obtained. Orientation may be determined to accuracies approaching $0.1^{\circ}$ of arc. Finally, a complete stress-strain analysis may be obtained from line profiles and intensities.

It was known 30 years ago that such information could be obtained; nevertheless, the divergent beam method was not widely used until about 1962 . In the past 5 years, new procedures allowing the investigator to obtain all of these data in a straightforward, rigorous fashion have been developed [3-10]. However, a drawback to the use of the divergent beam technique was the fact that the development of suitable instrumentation proceeded at a slow pace. This paper describes the design of a Kossel pattern generator (KPG) which enables the investigator to take

${ }^{1}$ Figures in brackets indicate the literature references at the end of this paper. full advantage of the divergent beam technique. The KPG is a self-contained instrument of modular design whose camera component meets all of the preferred design criteria described by Vieth and Yakowitz [11].

\section{Evolution of Kossel Instrumentation}

The first successful experimental demonstration of a divergent beam diffraction technique was made by Rutherford and Andrade in 1914. These workers used an external radium source of $\gamma$-rays and a cleaved rocksalt crystal as the target. From the photographically recorded diffraction pattern, they were able to determine the monochromatic radiation components of the source [12].

Three years later Seemann [13] developed a special wide angle tube which allowed $\mathrm{x}$ rays produced over a large area to converge and then to exit through a small circular aperture. In 1921, Gerlach [14] developed a system in which a fine beam of high energy electrons impinged upon a thin foil target. The $\mathrm{x}$ rays which were produced in this thin foil diverged through it as well as the specimen crystal which was placed in the opposite side of the foil. This was the forerunner of the modern capillary $x$-ray tube device which is still commercially marketed.

In 1929, Linnik [15] tried a novel approach using conventional x-ray equipment. A fine beam of monochromatic $\mathrm{x}$ rays was allowed to pass through a thin crystal. A given crystal volume was made to successively occupy all possible positions within a defined solid angle. The rigidly connected crystal-film assembly was slowly rotated around an axis which was parallel to the x-ray beam and which passed through the crystal while remaining perpendicular to the film. Simultaneously, the assembly was rocked about an axis orthogonal to this which passed through the crystal and intersected the rotation axis.

Kossel [16] designed a system in which the crystal of interest was made the anticathode of the x-ray 
tube. The system was maintained in vacuum and a set of apertures was used to produce a narrow beam of electrons. Later an electromagnetic lens was used to decrease the size of the beam of electrons striking the crystal. An aluminum window provided the exit port for the diffracted $x$ rays.

Borrman [17], using conventional x-ray equipment, employed a narrow beam of white radiation to cause fluorescence of the elements of a crystal. The resulting divergent secondary radiation produced the desired patterns. In this method, Laue spots appear in addition to the divergent beam pattern.

In addition to being cumbersome and complex, all divergent beam devices prior to 1951 suffered the handicap of relatively large sources of $x$-radiation. This led to several problems: a large primary source causes the Kossel lines to be broadened and impairs the inherent precision of the method; in addition, exposure times are prohibitively long $(24 \mathrm{hr}$ in some cases) and photographic contrast is poor. Furthermore, large single crystals are required to accommodate the large x-ray sources.

These problems are removed by the use of the electron probe microanalyzer. This instrument provides a micron sized source of $\mathrm{x}$ rays, as well as an optical microscope for viewing the area undergoing irradiation. Power densities five to ten times greater than those obtainable with earlier equipment are available. The value of the electron probe microanalyzer as a source for Kossel patterns was recognized by Castaing as soon as his first instrument was completed. He presented Kossel patterns in his Thesis which clearly demonstrated the superior characteristics of the microprobe in this area [18].

\section{Economics Applied to Modern Kossel Instrumentation}

All present day Kossel instrumentation utilizes a focused electron beam to excite the required microsource of $x$-radiation. While commercial Kossel camera attachments for electron probe microanalyzers represent the most readily available instrumentation, investigators have successfully prepared Kossel cameras for use in point-projection x-ray microscopes and in electron microscopes, possibly since all of these instruments provide convenient electron optics.

However, adapting such instrumentation to eiectron optical columns primarily designed for other purposes is usually inefficient. Consider a commercial electron probe microanalyzer as an example. Normally, when Kossel patterns are being prepared, equipment including x-ray spectrometers, (multichannel analyzer), scanning displays, and x-ray read out channels is inoperative. Furthermore, almost all Kossel cameras built for insertion into existing electron columns contain serious compromises with preferred design characteristics [11]. Hence, it is not only uneconomical to tie up a microprobe or electron microscope, but in addition, research may be hindered by the camera design.
Another important consideration is that the electron optical requirements for the vast majority of Kossel line based research are less stringent than those for electron probe microanalysis and electron or x-ray microscopy. A single electromagnetic lens combined with an inexpensive flat grid election gun makes an entirely satisfactory electron column.'A relatively inexpensive power supply can be used since gun voltage stability requirements are less demanding than for electron microanalysis or microscopy. The same is true for the single lens power supply needed. To complete the system (exclusive of the Kossel camera), a small vacuum system, vacuum gauges, suitable light optics, and a current meter whose range is 10 to 1000 nanoamperes are required. Finally, a modular design can be modified at will to incorporate improvements. On the other hand, it may prove difficult to modify an electron microprobe or microscope for many reasons. Based on the foregoing discussion, the decision was made to design and build a separate KPG.

\section{Design of the Kossel Pattern Generator}

\subsection{Basic Considerations}

Kossel patterns can be obtained either in transmission or in back reflection. In the transmission mode, the pattern is representative of the entire sample thickness traversed. Sample thicknesses are usually chosen for $\mu X=2, \mu$ being the linear $\mathrm{x}$-ray attenuation coefficient for the characteristic radiation of interest and $X$ being the thickness [19]. In back reflection, only regions near the surface are sampled so the specimen can be thick.

All of the information about orientation, lattice spacings and strain configuration can be obtained from either type of pattern. However, from detailed considerations of the two modes, we concluded that the transmission pattern is best for precise lattice spacing measurements. Back reflection is probably superior for most orientation studies [11]. Since much of our work is concerned with the study of small changes in lattice spacings as a function of applied stress or other perturbation, we decided to build the KPG suitable for work in the transmission mode. This choice only affects the camera module design. The electron beam column to be described can be used with a back reflection camera as well. The unit consists of (1) Kossel camera, (2) Light microscope, (3) Film cassette, (4) Electron beam column, (5) A suitable vacuum system. Figure 1 shows the assembled instrument.

\subsection{The Kossel Camera}

The most important design problem concerns the camera module. The remainder of the KPG is designed around the camera. The design concepts for a successful Kossel camera have been detailed previously [11]. They may be summarized as follows:

(1) It should be possible to locate inclusions, twins, 


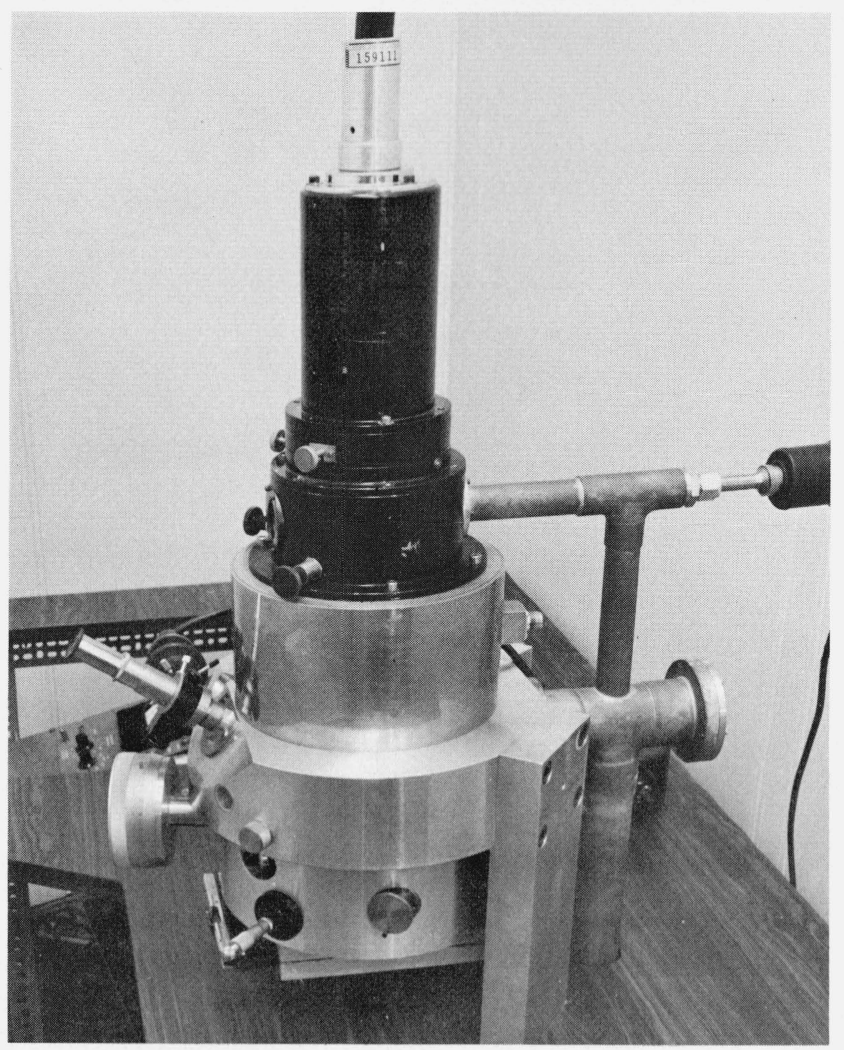

Figure 1. General view of the KPG fully assembled.

slip bands, grain boundaries, etc., and place them under the beam. This requires capabilities for movement of the specimen in orthogonal directions in its own plane as well as in a direction perpendicular to the specimen plane, and for an optical microscope to view the area of interest,

(2) To eliminate the distortion effects of geometric projection on the flat film, carry out orientation studies, and for almost all lattice spacing studies, the capability of orienting the specimen by means of a microgoniometer controlled from outside the vacuum system is necessary,

(3) The pattern center position must be found for almost all lattice spacing studies and as an origin for orientation studies. Therefore, one must be able to mark the position of the pattern center precisely,

(4) Small values of the specimen-to-film distance may cause contrast losses on the film while large values may increase exposure times to prohibitive values. A compromise is a specimen-to-film distance of 5 to $11 \mathrm{~cm}$,

(5) The angle between the important cubic poles $\langle 100\rangle$ and $\langle 111\rangle$ is about $55^{\circ}$ so that an $\mathrm{x}$-ray divergence angle of at least $56^{\circ}$ is desired on the film, and

(6) Convenience and control of operation are greatly increased by designing the camera so that the specimen is in the vacuum system but the film is in air.

The entire system was designed so that it could be dismantled and repaired with a minimum of inconvenience while still satisfying all of the design concepts listed. The camera is a separate module removable from the electron beam column by means of three bolts.

The stage allows translation of the specimen amounting to $1 / 2$ in in orthogonal directions. Each motion is spring loaded to minimize backlash and each is connected by means of vacuum shaft seals to a micrometer shaft. Thus, the coordinates of a given point on the specimen can be found and recorded so that the point can be found again later. The vertical motion of the stage which makes it possible to focus the optical microscope is controlled by a worm and wheel arrangement. The wheel has 230 teeth and advances a four lead thread $5 / 8$ in per revolution. This gives a rapid vertical motion but retains enough sensitivity to focus easily in the light microscope.

A two-axis goniometer is mounted on this mechanical stage. Each tilt motion can be controlled from outside the vacuum system. An indicating dial is used to index the angle of each axis. The tilt is controlled by a worm and a worm-gear; each tilt mechanism is spring loaded to minimize the effects of backlash. We were forced to use a small worm-gear in view of space limitations. This limited the number of teeth and hence the angular sensitivity of the goniometer to a minimum readable motion of about $0.2^{\circ}$. The goniometer may be tilted $\pm 12^{\circ}$ about each axis. At this angle the edge of the stage strikes the light optical objective lens.

The essential part of the goniometer design as well as of the vertical specimen motion is the linkage connecting the external control device to the actual moving part. Each linkage can move through an angle up to $15^{\circ}$, traverse a distance of $0.70 \mathrm{in}$, and rotate freely. This is accomplished by attaching small universal joints on the ends of standard sliding tubular linkage. In order to prevent galling of the sliding mechanism in vacuum, we lubricate each part with a suspension of $\mathrm{MoS}_{2}$ in diffusion pump oil. These linkage components are shown in place in figure 2.

Finding and marking the pattern center is carried out by a method devised by Ogilvie [20]. A small aperture at the specimen plane is brought to the coordinate point of the beam. Light from the hot filament is allowed to strike the film; the high voltage is switched off during this operation. A small spot is impressed at the pattern center by this means.

The specimen holder will accommodate a $3 / 4$ in diam sample. Holders are of brass and the specimen is sandwiched between two brass discs. A source foil for pseudo-Kossel studies may be placed on the top disc where it is not in thermal contact with the actual specimen. The entire assembly fits into an insulating ring; a lead wire connects to a vacuum electrical feedthrough. In this fashion, electron current from the specimen can be read on an appropriate electrometer.

For transmission studies, the specimen-to-film distance has little effect on the photographic contrast, which is controlled by the operating voltage, specimen thickness and crystal perfection [19]. We chose a specimen-to-film distance of $12 \mathrm{~cm}$ as a compromise be- 


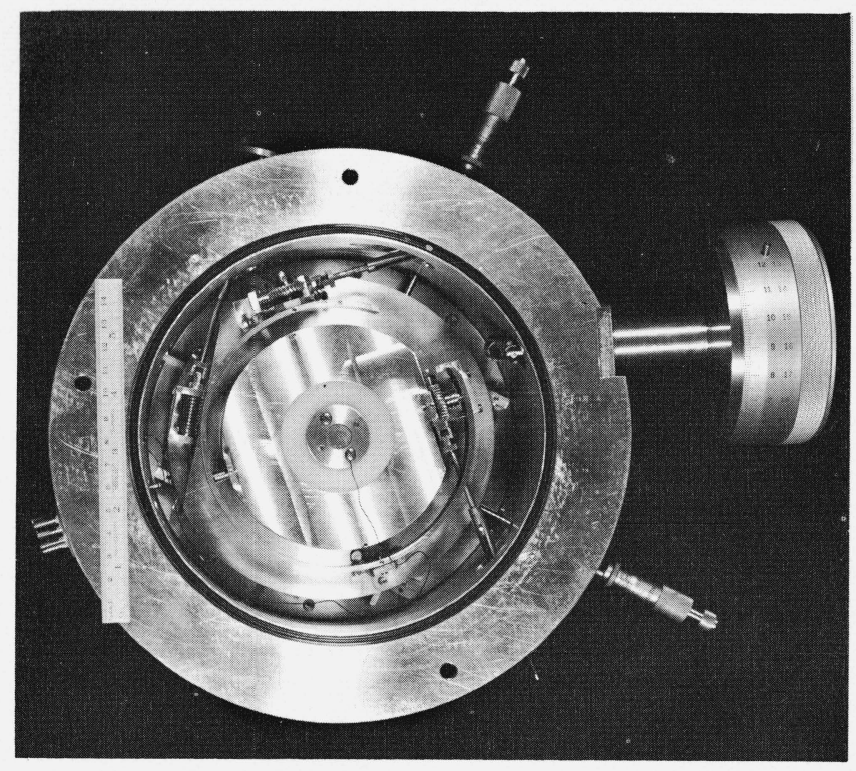

FigURE 2. Kossel camera showing linkage for $X-Y-Z$ and tilt motions. Specimen in place.

tween exposure time, angular divergence to be recorded on the film and Kossel line distribution on the pattern. With this distance, a naximum divergence angle of $70^{\circ}$ is recorded on the film. This value is almost always satisfactory for obtaining Kossel pattern geometry suitable for precisely determining lattice spacings [9]. Furthermore, sufficient information is provided for indexing and orientation determination of most crystals with the $70^{\circ}$ divergence angle.

Patterns are recorded on standard $5 \times 7$ in $x$-ray film or glass plates. The film cassette is an ordinary plate-holder which fits into an aluminum frame bolted onto the camera bottom. The film is in air so that many exposures may be taken without disturbing the crystal or the vacuum. This is accomplished by placing a polyester window 5 mils thick directly below the specimen (fig. 3). The window diameter is $17 / 8$ in. The window sits in a removable cap and is sealed by $\mathrm{O}$-rings inside and outside. Window failure is rare provided normal care is exercised.

\subsection{Optical Microscope}

We found refracting optics viewing the specimen at a $35 \mathrm{deg}$ angle to the horizontal by means of appropriately placed mirrors to be unsatisfactory. Image distortion was severe and the focusing characteristics Itoo critical for convenient use. Therefore, the system chosen consists of a modified commercially available reflecting objective $(50 \times$, numerical aperture 0.56 , working distance $5.5 \mathrm{~mm}$ ), vertical illuminator and ocular. The objective is placed between the pole piece of the electromagnetic lens and the specimen. Controls are provided so that the axis of the light optical objective can be made to coincide with the axis of the electron beam. Figure 4 shows the light optical system.

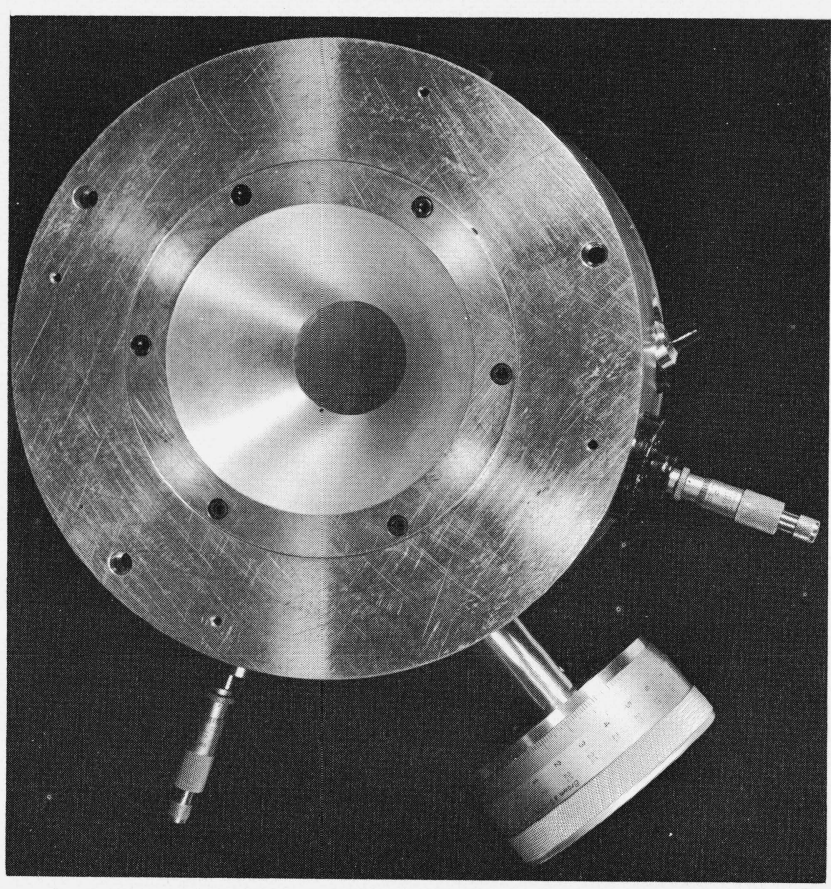

FIGURE 3. Bottom view of Kossel camera showing polyester window with specimen visible beyond.

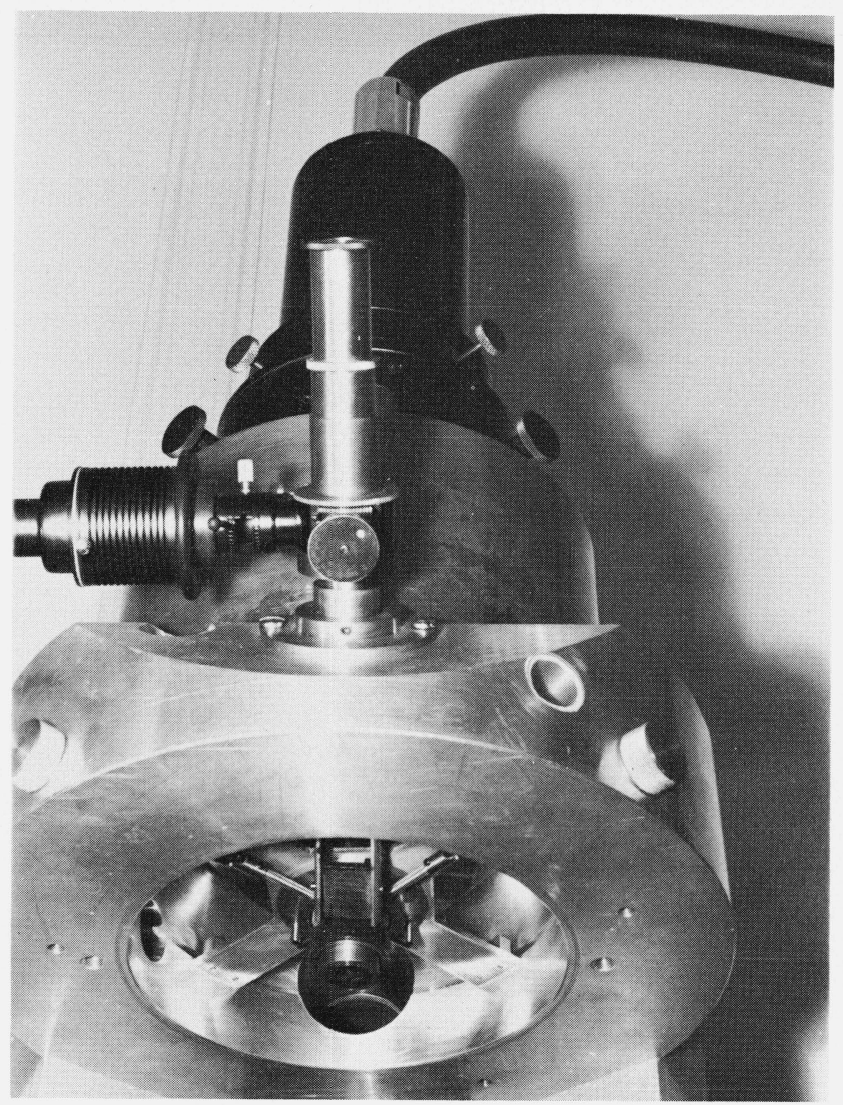

FIGURE 4. View of the column showing the light optical system: objective, transfer mirror, centering devices, light source, vertical illuminator and ocular. 


\subsection{The Electron Beam Column}

The column design is based on the general discussion of single lens systems given by Cosslett, Nixon, and Pearson [21]. A magnetic lens was chosen because its spherical aberration constant, $C_{s}$, is smaller than that of a corresponding electrostatic lens. The smallest beam that can be obtained with an appropriately placed aperture, which accepts the full beam current, requires $C_{s}$ to be minimized. In order to accommodate the light optical system, the specimen must be placed 1.8 in $(4.6 \mathrm{~cm})$ below the nose of the lens. This results in a focal length of $5 \mathrm{~cm}$ for the lens. This long focal length gives a value of $C_{s}$ equal to $67 \mathrm{~cm}$ with the asymmetric pole piece geometry of our lens. Nevertheless, calculations showed that a final beam diameter of about $1 \mu$ could be obtained at the specimen plane. This required a $25 \mu$ diam source to be limited by an aperture to $4.6 \times 10^{-4}$ radians. Placing an aperture $100 \mu$ in diameter some $5 \frac{1 / 2}{2}$ in $(14 \mathrm{~cm})$ below the electron gun filament achieved the desired result.

The electron gun chosen is a commercially available flat grid cap type usually supplied with small electron microscopes. It is mounted on the axis of the lens. The gun bias is operated nearly at the cutoff point in order to reduce beam angle and to operate where the source brightness is greatest. The filament is a hairpin loop of $8 \mathrm{mil}$ diam tungsten wire. Centering of the filament within the grid cap as well as the filament tip height adjustment with respect to the cap can only be carried out with the gun removed from the column.
This has not proved to be a great liability. We have provided controls for centering the gun assembly and the aperture with respect to the axis of the electromagnetic lens.

The power supply is capable of providing a voltage between 5 and $50 \mathrm{kV}$. The filament heater, bias resistance and lens current supply complete the requirements for obtaining the electron beam. A suitable vacuum system consisting of forepump, 2 -in diffusion pump, appropriate valves, gauges and piping and a cold-baffle completes the basic column. A circulating refrigerant cold trap is used so as to eliminate recharging problems.

The electron beam is monitored by an indicating nanoammeter (range $1-10,000 \mathrm{nA}$ ) equipped with a charge integrator. This device is connected to the specimen; the specimen is isolated from ground so that the electrometer can read the electron charge "absorbed" in the specimen. This current, generated by the electron flow, is called the specimen current. Several advantages result from being able to monitor the specimen current. First, we use it to know when the gun is saturated. After electron beam alinement within the column, variation of the specimen current is a measure of system instability. It is also helpful to use the integrated charge to obtain the proper photographic exposure. This is done by using the equations relating exposure and incident electrons given by Yakowitz and Vieth [22]. The advantage of this procedure is that slight variations in the specimen current can be tolerated by using the total charge.

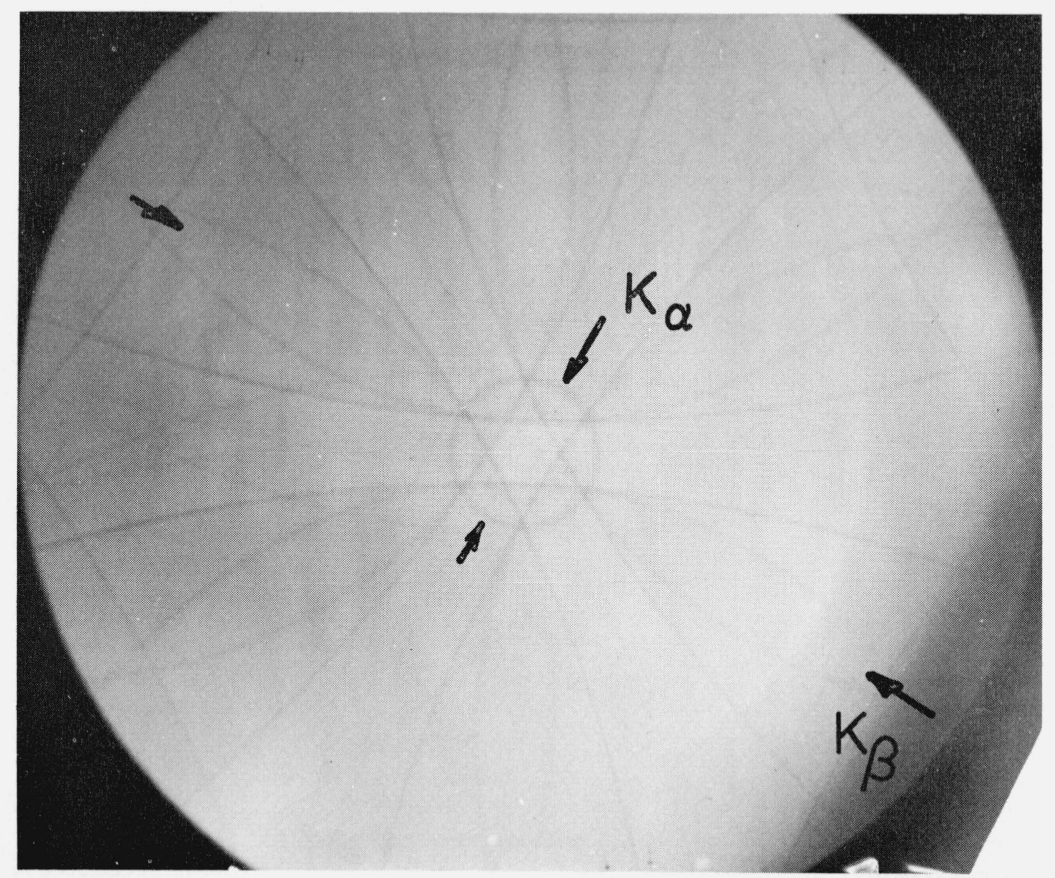

Figure 5. Transmission Kossel pattern of $\mathrm{Be}-4.4$ w/o $\mathrm{Cu}$ alloy using $\mathrm{Co}-\mathrm{K}$ radiation. The position of the $K \beta$ circle, only faintly visible on the print, is shown by arrows. The $K \alpha$ circle is also marked. 


\section{Performance}

The KPG has been used to study $\mathrm{SrTiO}_{3}$, nearly perfect silicon, Fe-3 wt. pct. Si alloy, indium, beryllium and beryllium alloys. The case of Be-4.4 wt. pet. $\mathrm{Cu}$ will serve as a brief example. This alloy is interesting since conventional $x$-ray methods for determining its lattice constants are difficult to apply. Furthermore the alloy has a hexagonal close-packed structure; traditionally, the Kossel method has been applied to cubic structures.

Witt et al., determined a general method for the solution of lattice spacings in noncubic structures by the Kossel technique [10]. This method requires one, two, or even three different crystal orientations for success. Thus, the necessity for a goniometer and the ability to take many photographs rapidly are underlined.

The method was applied to the Be-4.4 wt. pct. Cu alloy: Co-K radiation was used in the pseudo-Kossel experiment (for details, see [10]). All patterns were prepared in the KPG; orientation was carried out so that the $\langle 00.1\rangle$ pole would be exactly perpendicular to the film. The resulting pattern is shown in figure 5 . By measuring the ratio of the $K \alpha_{1}$ to $K \beta$ circle diameters, the lattice constants of the alloy were determined as $c=3.5908 \AA$ and $a=2.2838 \AA$ giving $c / a=1.57$. The entire experiment to obtain figure 5 required less than $2 \mathrm{hr}$. Without the goniometric capabilities, the experiment could not have been properly performed. Without the capability for preparing many photographs without disturbing the crystal or the vacuum, we estimate the time to obtain figure 5 at about 10 working hr.

Special thanks are due to L. Schneider and C. Taylor of the NBS Instrument Shops for their cooperation in the manufacture of the KPG.

\section{References}

[1] R. W. James, The Optical Principles of the Diffraction of $\mathrm{X}$ rays, Vol. II of The Crystalline State, p. 439, L. Bragg, ed., (G. Bell and Sons Ltd, London, 1950).

[2] T. Imura, S. Weissmann, and J. J. Slade, Jr., A study of agehardening of $\mathrm{Al}-3.85 \% \mathrm{Cu}$ by the divergent $\mathrm{x}$-ray beam method, Acta Cryst. 15, 786 (1962).

[3] R. E. Hanneman, R. E. Ogilvie, and A. Modrzejewski, Kossel line studies of irradiated nickel crystals, J. Appl. Phys. 33, $1429(1962)$
[4] E. T. Peters and R. E. Ogilvie, X-ray orientation and diffraction studies by Kossel lines, Trans. AIME 233, 89 (1965).

[5] J. J. Slade, Jr., S. Weissmann, K. Nakajima, and M. Hirabayashi, Stress-strain analysis of single cubic crystals and its application to the ordering of CuAuI, J. Appl. Phys. 35, 3373 (1964).

[6] T. Ellis, L. F. Nanni, A. Shrier, S. Weissmann, G. E. Padawer, and N. Hosokawa, Strain and precision lattice parameter measurements by the x-ray divergent beam method I, J. Appl. Phys. 35, 3364 (1964).

[7] M. Bevis and N. Swindells, The determination of the orientation of micro-crystals using a back-reflection technique and an electron probe microanalyser. Phys. Stat. Sol. 20, 197 (1967).

[8] P. Gielen, H. Yakowitz, D. Ganow, and R. E. Ogilvie, Evaluation of Kossel microdiffraction procedures: The cubic case, J. Appl. Phys. 36, 773 (1965).

[9] H. Yakowitz, Precision of Cubic Lattice Parameter Measurements by the Kossel Technique, in The Electron Microprobe, p. 417, Proc. Symp. Electrochem. Soc., Washington, D.C. $1964-$ T. D. McKinley, K. F. J. Heinrich and D. B. Wittry, eds. (John Wiley \& Sons, Inc., New York, N.Y., 1966).

[10] F. Witt, H. Yakowitz, and D. L. Vieth, A method for determining lattice spacings in non-cubic materials by the Kossel technique, to be published.

[11] D. L. Vieth and H. Yakowitz, Design considerations for a Kossel microdiffraction camera, Rev. Sci. Instr. 3 7, 206 (1966).

[12] E. Rutherford and E. N. da C. Andrade, The spectrum of the penetrating $\gamma$ rays from radium $B$ and radium C., Phil. Mag. [6] 28, 263 (1914).

[13] H. Seemann, The reflection optics of x-rays by crystal structure surfaces II, Ann. Phys. 53, 461 (1917).

[14] W. Gerlach, Crystal lattice studies using x-rays and a simple x-ray tube, Physik. Zeitschr. 22, 557 (1921).

[15] W. Linnik, Method of determining the position of the symmetry axis of a crystal by means of $x$-rays, Nature (Lond.) 124, 946 (1929).

[16] W. Kossel, X-ray interferences from (x-ray) sources within the lattice, Ergebn. exakt. Naturw. 16, 295 (1937).

[17] G. Borrmann, Concerning the interferences caused by x-ray excitation of (x-ray) sources within the lattice, Ann. Phys. 27, 669 (1936).

[18] R. Castaing, Application of electron probes to local chemical and crystallographic analysis, Thesis to University of Paris (1951).

[19] H. Yakowitz, Effect of sample thickness and operating voltage on the contrast of Kossel transmission photographs, J. Appl. Phys. 37, 4455 (1966).

[20] R. E. Ogilvie, private communication (1964).

[21] V. E. Cosslett, W. C. Nixon, and H. E. Pearson, Improvements in the Point Projection X-ray Microscope, in X-ray Microscopy and Microradiography (Proc. Symp. Cavendish Lab., Cambridge, England, 1956-V. E. Cosslett, A. Engström, and H. H. Pattee, Jr., eds.) (Academic Press, New York (1957) p. 96.)

[22] H. Yakowitz and D. L. Vieth, Exposure Time Relations for Kossel Microdiffraction Photographs, J. Res. NBS 69C (Engr. and Instr.) No. 3, 213 (1965).

(Paper 71C4-262) 